\title{
FOLIACIONES DICRÍTICAS SIN SINGULARIDADES DESPUÉS DE UN BLOW-UP
}

\author{
Renato Benazic Tomé
}

Resumen.- En el presente trabajo estudiamos foliaciones definidas por campos vectoriales holomorfos con singularidad aislada en el origen del tipo dicrítico, cuyo transformado estricto no presenta singularidades y que satisface la $\tau$-condición. Probaremos que esta familia es abierta y densa y daremos formas locales de todos los puntos del divisor.

Palabras claves: Foliaciones singulares por curvas, Foliaciones con singularidades Dicríticas, Campos Vectoriales Holomorfos. Reducción de singularidades.

\section{DICRITIC FOLIATION WITHOUT SINGULARITIES AFTER A BLOW-UP}

\begin{abstract}
In this paper we are going to study foliations defined by holomorphic vector fields with isolated singularity in the origin with dicritic type, which strict transformed doesn't present singularities and satisfies the $\tau$-condition. We will prove that this family is open and dense; also we will give local forms of every point of the divisor.
\end{abstract}

Key words: Singular foliations by curves, Foliations with dicritic singularities, Holomorphic vector fields. Reduction of singularities.

\section{INTRODUCCIÓN}

Sea $Z=\sum_{j=1}^{n} Z_{j} \frac{\partial}{\partial z_{j}}$ un campo vectorial holomorfo definido en una vecindad abierta $U \subseteq \mathbb{C}^{n}$ del $0 \in \mathbb{C}^{n}$. La Ecuación Diferencial Ordinaria asociada a $Z$ es el sistema

$$
\mid \begin{array}{ccc}
z_{1}^{\prime} & = & Z_{1}\left(z_{1}, \ldots, z_{n}\right) \\
z_{2}^{\prime} & = & Z_{2}\left(z_{1}, \ldots, z_{n}\right) \\
\vdots & \vdots & \vdots \\
z_{n}^{\prime} & = & Z_{n}\left(z_{1}, \ldots, z_{n}\right)
\end{array}
$$

${ }^{1}$ UNMSMS, Facultad de Ciencias Matemáticas, Instituto de Matemática y Ciencias Afines (IMCA). 
en donde $T \in \mathbb{C}$ y $z_{j}^{\prime}=\frac{d z_{j}}{d T}$. Las soluciones de (1) definen una foliación singular por curvas complejas del abierto $U$, la que denotaremos por $\mathcal{F}_{Z}$, sus elementos son llamados hojas. Una parte sumamente importante de la Teoría local de las Ecuaciones Diferenciales Ordinarias consiste en determinar el comportamiento geométrico de las soluciones (1) (es decir las hojas de $\mathcal{F}_{Z}$ ) en una vecindad del origen. En el presente trabajo, vamos a dar las formas locales de una familia de campos vectoriales holomorfos $Z$ con singularidad aislada y cuyo transformado estricto no presenta singularidades. Probaremos que esta familia es suficientemente grande, en el sentido que es abierta y densa.

\section{ALGUNAS DEFINICIONES}

A lo largo del presente trabajo, usaremos frecuentemente la notación de los multiíndices. Un multi-índice es una $n$-upla de números enteros no negativos $Q=\left(q_{1}, \ldots, q_{n}\right)$, su norma $|Q|$ se define como $|Q|=q_{1}+\cdots+q_{n}$ y si $z=\left(z_{1}, \ldots, z_{n}\right) \in \mathbb{C}^{n}$ entonces denotaremos $z^{Q}=z_{1}^{q_{1}} \cdots z_{n}^{q_{n}}$.

Sea $Z=\sum_{j=1}^{n} Z_{j} \frac{\partial}{\partial z_{j}}$ un campo vectorial holomorfo definido en una vecindad abierta $U \subseteq \mathbb{C}^{n}$ del $0 \in \mathbb{C}^{n}$. Usando las notaciones anteriores, como cada $Z_{j}$ es una función holomorfa de varias variables complejas entonces $Z_{j}$ admite un desarrollo en serie de Taylor en una vecindad del 0, i.e.

$$
Z_{j}(z)=\sum_{|Q|=1}^{\infty} a_{j, Q} z^{Q}=\sum_{k=1}^{\infty}\left(\sum_{|Q|=k} a_{j, Q} z^{Q}\right)=\sum_{k=1}^{\infty} Z_{j}^{k}(z)
$$

en donde los $Z_{j}^{k}(z)=\sum_{|Q|=k} a_{j, Q} z^{Q}$ son polinomios homogéneos de grado $k$ en las variables complejas $z_{1}, \ldots, z_{n}$.

El orden de $Z_{j}$ en el 0 , denotado por ord ${ }_{0}\left(Z_{j}\right)$ es, por definición, el menor número entero positivo $\nu_{j}$ tal que $Z_{j}^{1} \equiv 0, \ldots Z_{j}^{\nu_{j}-1} \equiv 0$ y $Z_{j}^{\nu_{j}} \not \equiv 0$. El orden del campo $Z$ en 0 es por definición, el mínimo de los órdenes (en cero) de sus coordenadas, es decir

$$
\operatorname{ord}_{0}(Z)=\min \left\{\operatorname{ord}_{0}\left(Z_{1}\right), \ldots, \operatorname{ord}_{0}\left(Z_{n}\right)\right\}
$$


Observe que

$$
\operatorname{ord}_{0}(Z)=\nu \Longleftrightarrow Z=Z_{\nu}+Z_{\nu+1}+\cdots=\sum_{k \geq \nu} Z_{k}
$$

donde $Z_{k}$ es el campo polinomial homogéneo de grado $k$ cuyas coordenadas son $Z_{j}^{k}$, es decir

$$
Z_{k}=\sum_{j=1}^{n} Z_{j}^{k} \frac{\partial}{\partial z_{j}}
$$

Observe también que $Z_{1}$ es un campo polinomial homogéneo de grado uno, es decir, un campo lineal que coincide con la derivada (compleja) de $Z$ en $0 \in \mathbb{C}^{n}$ y al cual se le puede asociar su matriz Jacobiana (compleja)

$$
Z^{\prime}(0)=\left[\begin{array}{ccc}
\frac{\partial Z_{1}}{\partial z_{1}}(0) & \cdots & \frac{\partial Z_{1}}{\partial z_{n}}(0) \\
\vdots & & \vdots \\
\frac{\partial Z_{n}}{\partial z_{1}}(0) & \cdots & \frac{\partial Z_{n}}{\partial z_{n}}(0)
\end{array}\right]
$$

$Z^{\prime}(0)$ es llamado parte lineal del campo $Z$.

Decimos que $0 \in U$ es un punto singular de $Z$ si y sólo si $\operatorname{ord}_{0}(Z) \geq 1$, caso contrario decimos que 0 es un punto regular de $Z$. El conjunto de todos los puntos singulares de $Z$ será denotado por $\operatorname{Sing}(Z)$.

Es bien sabido que si $0 \in U$ es un punto regular del campo (es decir $Z(p) \neq 0$ ) entonces el teorema del flujo tubular (ver [3], [12]) nos da un comportamiento local satisfactorio de la foliación en la vecindad del punto 0 (las curvas solución pueden ser enderezadas). En cambio si $0 \in \operatorname{Sing}(Z)$ el comportamiento local de la foliación $\mathcal{F}_{Z}$ alrededor del cero es mucho más interesante y su complejidad esta íntimamente relacionada a que tan grande es el $\operatorname{ord}_{0}(Z)$.

Si $\operatorname{ord}_{0}(Z)=1$ entonces el campo $Z$ tiene parte lineal no nula y para conocer el comportamiento geométrico de $\mathcal{F}_{Z}$ en una vecindad del origen, pueden usarse los resultados de linealización de Poincaré y Siegel o el Teorema de Dulac.

En cambio, si $\operatorname{ord}_{0}(Z)>1$ entonces se usan técnicas de reducción del orden de la singularidada través del blow-up, concepto que pasamos a explicar brevemente.

Sea $E: \widetilde{U} \rightarrow U$ el blow-up centrado en $0 \in \mathbb{C}^{n}$, entonces es conocido que existe una única manera de extender el pull-back $E^{*}\left(\mathcal{F}_{Z}-\{0\}\right)$ a una foliación analítica $\widetilde{\mathcal{F}}_{Z}$ sobre una vecindad del divisor $D=\mathbb{C} P(n-1)=E^{-1}(0) \subseteq \widetilde{U}$ de tal manera que su conjunto singular sea de codimensión mayor que o igual a 2 . En este caso decimos que $\widetilde{\mathcal{F}}_{Z}$ es el transformado estricto de $\mathcal{F}_{Z}$ por el Blow-up $E$. 
Decimos que $0 \in \mathbb{C}^{n}$ es una singularidad no dicrítica de $\mathcal{F}_{Z}$ (o de $Z$ ) si y sólo si el divisor $D$ es invariante por $\widetilde{\mathcal{F}}_{Z}$. En caso contrario, decimos que $0 \in \mathbb{C}^{n}$ es una singularidad dicrítica de $\mathcal{F}_{Z}$.

En dimensión $n=2$ existe un famosos resultado debido a Seidenberg (ver [10], [13]) el cual establece que después de un número finito de blow up, el transformado estricto correspondiente sólo tiene singularidades con parte lineal no nula (orden 1). Este resultado es conocido como teorema de reducción de singularidades.

En dimensión $n \geq 3$ no se conoce aún un teorema general de de reducción de singularidades, aunque existen algunos resultados parciales: Cano (dimensión 3 (ver [5]), Camacho, Cano, Sad, (dimensión $n$ cualquiera pero con hipótesis de singularidad no dicrítica absolutamente aislada, ver [6]), Benazic (dimensión $n$ cualquiera pero con hipótesis de singularidad absolutamente aislada, ver [1]), etc.

En el presente trabajo, estudiaremos una familia de campos vectoriales holomorfos con singularidad aislada en el origen y cuyo transformado estricto no presenta singularidades.

\section{CAMPOS CUYO TRANSFORMADOS ESTRICTOS NO TIENEN SINGULARIDADES}

Sea $Z$ campo vectorial holomorfo con singularidad aislada en el $0 \in \mathbb{C}^{n}$ (es decir que existe una vecindad del origen tal que 0 es la única singularidad de $Z$ ) tal que $\operatorname{ord}_{0}(Z)=\nu$, luego $Z=Z_{\nu}+Z_{\nu+1}+Z_{\nu+2}+\cdots$. Si 0 es singularidad dicrítica de $Z$ entonces no es difícil probar que las siguientes condiciones son equivalentes (ver [2]):

1. $0 \in \mathbb{C}^{n}$ es una singularidad de $Z$.

2. $z_{j} Z_{\nu}^{i}-z_{i} Z_{\nu}^{j}=0, \forall 1 \leq i \neq j \leq n$.

3. $Z_{\nu}=P_{\nu-1} R$ donde $P_{\nu-1}$ es un polinomio homogéneo de grado $\nu-1$ y $R=\sum_{j=1}^{n} z_{j} \frac{\partial}{\partial z_{j}}$ es el campo radial.

Más aún, si cualquiera de las tres condiciones anterioreses verdadera, entonces $\operatorname{Sing}\left(\widetilde{\mathcal{F}}_{Z}\right)$ es el conjunto de todos los puntos $\left[z_{1} ; \ldots ; z_{n}\right] \in D$ que son soluciones del siguiente sistema 
de $\frac{(n-1) n}{2}+1$ ecuaciones homogéneas:

$$
\mid \begin{aligned}
& P_{\nu-1}(z)=0 \\
& z_{j} Z_{\nu}^{i}-z_{i} Z_{\nu}^{j}=0, \quad 1 \leq i<j \leq n
\end{aligned}
$$

Se sigue que $\operatorname{Sing}\left(\widetilde{\mathcal{F}}_{Z}\right)=\emptyset$ si y sólo si $z=0 \in \mathbb{C}^{n}$ es la única solución del sistema anterior. Denotaremos por $\mathcal{D}_{\nu}^{n}$ al conjunto de todos los campos vectoriales holomorfos $Z$ definidos en una vecindad del origen de $\mathbb{C}^{n}$ tales que $0 \in \mathbb{C}^{n}$ sea una singularidad dicrítica aislada de $Z, m_{0}(Z)=\nu$ y $\operatorname{Sing}\left(\widetilde{\mathcal{F}}_{Z}\right)=\emptyset$. Observe que los elementos de $\mathcal{D}_{\nu}^{n}$ son campos vectoriales holomorfos que se desingularizan después de un blow-up. Este es un caso particular del Teorema de Reducción de singularidades de Seidenberg.

En el caso de dimensión $n=2$, los elementos de $\mathcal{D}_{\nu}^{n}$ fueron estudiados y clasificados por M. Klughertz (ver [8]).

\section{4. ÍNDICE DE INTERSECCIÓN Y EL ORDEN DE TANGENCIA}

Para cada $Z \in \mathcal{D}_{\nu}^{n}, Z=P_{\nu-1} R+\sum_{k \geq \nu+1} Z_{k}$, definimos la hiperficie algebraica $T$ sobre $D=\mathbb{C} P(n-1)$ como

$$
T=\left\{\left[z_{1} ; \ldots ; z_{n}\right] \in D ; P_{\nu-1}\left(z_{1}, \ldots, z_{n}\right)=0\right\}
$$

Vamos a probar que si $p \notin T$ entonces la hoja de la foliación transformada estricta $\tilde{\mathcal{F}}_{Z}$ que pasa por $p$ es transversal a $D$. Para ello introduciremos el concepto de índice de intersección.

Sea $p \in D$, sabemos que $p$ es punto regular de $\widetilde{Z}$, sea $\widetilde{L}$ la hoja de $\widetilde{\mathcal{F}}_{Z}$ que pasa por $p$. Desde que $D$ y $\widetilde{L}$ son subvariedades analíticas de $\widetilde{U}$ de dimensiones complementarias y $p \in D \cap \widetilde{L}$, podemos definir el índice de intersección $i_{p}(D, \widetilde{L})$ de la hoja $\widetilde{L}$ con el divisor $D$ en el punto $p$. En efecto, podemos suponer sin pérdida de generalidad que $p$ esta en la carta de $\widetilde{U}$ tal que el blow-up se expresa como

$$
E\left(y_{1}, \ldots, y_{n}\right)=\left(y_{1} y_{n}, \ldots, y_{n-1} y_{n}, y_{n}\right)=\left(z_{1}, \ldots, z_{n}\right)
$$


En esta carta $\widetilde{\mathcal{F}}_{Z}$ es generada por el campo vectorial holomorfo $\widetilde{Z}=\sum_{i=1}^{n} \widetilde{Z}_{i} \frac{\partial}{\partial y_{i}}$, donde

$$
\mid \begin{aligned}
& \widetilde{Z}_{i}\left(y_{1}, \ldots, y_{n}\right)=\sum_{k \geq \nu} y_{n}^{k-\nu}\left[Z_{k+1}^{i}(\widehat{y})-y_{i} Z_{k+1}^{n}(\widehat{y})\right], \quad 1 \leq i \leq n-1 \\
& \widetilde{Z}_{n}\left(y_{1}, \ldots, y_{n}\right)=P_{\nu-1}(\widehat{y})+\sum_{k \geq \nu} y_{n}^{k-\nu} Z_{k}^{n}(\widehat{y})
\end{aligned}
$$

e $\widehat{y}=\left(y_{1}, \ldots, y_{n-1}, 1\right)$. Desde que $p=\left(\left(y_{1}^{0}, \ldots ., y_{n-1}^{0}, 0\right)\right.$ es un punto regular de $\widetilde{\mathcal{F}}_{Z}$, la hoja $\widetilde{L}$ puede ser localmente parametrizada por una función analítica

$$
\alpha=\left(\alpha_{1}, \ldots, \alpha_{n}\right):\left(D_{\epsilon}, 0\right) \rightarrow(\widetilde{L}, p)
$$

(donde $\left.D_{\epsilon}=\{t \in \mathbb{C} ;|t|<\epsilon\}\right)$ tal que

$$
\mid \begin{aligned}
& \alpha^{\prime}(t)=\widetilde{Z}(\alpha(t)) \\
& \alpha(0)=p
\end{aligned}
$$

Desde que $D=\left\{y_{n}=0\right\}$, definimos

$$
i_{p}(D, \widetilde{L})=\operatorname{ord}_{0}\left(\alpha_{n}\right)
$$

es decir

$$
i_{p}(D, \widetilde{L})=m \text { si y sólo si } \alpha_{n}(t)=t^{m} \xi_{n}(t)
$$

donde $\xi_{n}$ es una función analítica tal que $\xi_{n}(0) \neq 0$.

Es claro que la definición anterior es independiente de la elección de la parametrización $\alpha$ y que $i_{p}(D, \widetilde{L}) \geq 1, \forall p \in D$.

Se prueba que el índice de intersección es un invariante topológico (ver [2]) y puede ser geométricamente interpretado como el número de puntos de intersección de $L$ con una pequeña translación $\left\{y_{n}=\delta\right\}$ del divisor $D$ (ver [7]).

Existe otra definición equivalente del índice de intersección (ver [9], [4]). En efecto, 
sabemos que siempre es posible encontrar una función $\beta$ sobre $U$ tal que

$$
\begin{aligned}
& \text { a) } \beta^{-1}(0)=D \\
& \text { b) } 0 \in \mathbb{C} \text { es valor regular de } \beta
\end{aligned}
$$

Dado $\widetilde{Z}=\sum_{i=1}^{n} \widetilde{Z}_{i} \frac{\partial}{\partial y_{i}}$, definimos $\widetilde{Z}^{0}(\beta)=\beta, \widetilde{Z}^{1}(\beta)=\sum_{i=1}^{n} \widetilde{Z}_{i} \frac{\partial \beta}{\partial y_{i}}$ y para $k \geq 1$ definimos $\widetilde{Z}^{k+1}(\beta)$ inductivamente por $\widetilde{Z}^{1}\left(\widetilde{Z}^{k+1}(\beta)\right)$.

Definición 4.1 Sea $\beta$ una función satisfaciendo (5) y suponga que $Z \in \mathcal{D}_{\nu}^{n}$. Para $k \in \mathbb{N}$ definimos el conjunto de tangencia de orden $k, \Gamma_{k}(\widetilde{Z})$, como el conjunto de todos los puntos $p \in D$ tales que

$$
\beta(p)=\widetilde{Z}^{1}(\beta)(p)=\cdots=\widetilde{Z}^{k}(\beta)(p)=0 \quad \text { y } \quad \dot{\widetilde{Z}}^{k+1}(\beta)(p) \neq 0
$$

Es fácil chequear que la definición de los conjuntos $\Gamma_{k}(\widetilde{Z})$ es independiente de la elección de la función $\beta$ satisfaciendo (5). El siguiente resultado justifica la razón por la cual los conjuntos $\Gamma_{k}(\widetilde{Z})$ reciben el nombre de conjunto de tangencia de orden $k$.

Proposición 4.1 Con las notaciones anteriores, tenemos

$$
p \in \Gamma_{k}(\widetilde{Z}) \text { si y sólo si } i_{p}(D, \widetilde{L})=k+1
$$

Prueba. En la carta de $\widetilde{U}$ tal que el blow-up se expresa como

$$
E\left(y_{1}, \ldots, y_{n}\right)=\left(y_{1} y_{n}, \ldots, y_{n-1} y_{n}, y_{n}\right)=\left(z_{1}, \ldots, z_{n}\right)
$$

el divisor es dado por $D=\left\{y_{n}=0\right\}$, por lo tanto tomamos $\beta$ como $\beta\left(y_{1}, \ldots, y_{n}\right)=y_{n}$. Sea $p \in D$ y $\widetilde{L}$ la hoja de $\tilde{\mathcal{F}}_{Z}$ que pasa por $p$, para $\alpha$ definido como (3) tenemos que $\alpha_{n}(t)=\beta(\alpha(t))=\widetilde{Z}^{0}(\beta)(\alpha(t))$. Suponga, por inducción, que $\alpha_{n}^{(k)}(t)=\widetilde{Z}^{k}(\beta)(\alpha(t))$, 
entonces

$$
\alpha_{n}^{(k+1)}(t)=\sum_{i=1}^{n} \frac{\partial \widetilde{Z}^{k}(\beta)}{\partial y_{i}}(\alpha(t)) \alpha_{i}^{\prime}(t)=\sum_{i=1}^{n} \frac{\partial \widetilde{Z}^{k}(\beta)}{\partial y_{i}}(\alpha(t)) \widetilde{Z}_{i}(\alpha(t))=\widetilde{Z}^{k+1}(\beta)(\alpha(t))
$$

Concluímos que $\alpha_{n}^{(k)}(0)=\widetilde{Z}^{k+1}(\beta)(p), \forall k \in \mathbb{N}$. Por tanto

$$
\begin{aligned}
p \in \Gamma_{k}(\widetilde{Z}) & \Longleftrightarrow \tilde{Z}^{j}(\beta)(p)=0 \quad(0 \leq j \leq k) \quad \text { y } \quad \widetilde{Z}^{k+1}(\beta)(p) \neq 0 \\
& \Longleftrightarrow \alpha_{n}^{(j)}(0)=0 \quad(0 \leq j \leq k) \quad \text { y } \quad \alpha_{n}^{(k+1)}(0) \neq 0 \\
& \Longleftrightarrow i_{p}(D, \widetilde{L})=k+1
\end{aligned}
$$

De la proposición anterior se desprende que los $\Gamma_{k}(\widetilde{Z})$ son subconjuntos de $D$ disjuntos dos a dos y su unión es igual a $D$. Observe que dado $p=\left(y_{1}^{0}, \ldots, y_{n-1}^{0}, 0\right) \in D$, de (2) y (3) se tiene que

$$
\alpha_{n}^{\prime}(0)=P_{\nu-1}\left(y_{1}^{0}, \ldots, y_{n-1}^{0}, 1\right)
$$

Por tanto

$$
i_{p}(D, \widetilde{L})=1 \Longleftrightarrow p \in D-T \Longleftrightarrow \widetilde{L} \text { es transversal a } D
$$

Concluímos que $\Gamma_{0}(\widetilde{Z})=D-T$ tal como afirmamos al inicio de la sección. Por esta razón $T$ recibe el nombre de hiperficie de tangencia.

Para caracterizar los puntos de tangencia de orden $k \geq 1$, introducimos las hiperficies algebraicas

$$
S^{(k)}=\left\{p=\left[z_{1} ; \ldots ; z_{n}\right] \in D ; \widetilde{Z}^{k+1}(\beta)(p)=0\right\}
$$

Observe que para $k=0$, un fácil cálculo demuestra que $S^{(0)}=T$.

Proposición 4.2 Sea $k \in \mathbb{Z}^{+} y p \in S^{(0)} \cap S^{(1)} \cap \cdots \cap S^{(k-1)}$, entonces

$$
i_{p}(D, \widetilde{L})=k+1 \text { si y sólo si } p \notin S^{(k)}
$$

Prueba. Se sigue directamente de la Definición (4.1), la Proposición (4.1) y teniendo en cuenta la definición de las hiperficies algebraicas $S^{(i)}$. 
Corolario. Para cualquier $Z \in \mathcal{D}_{\nu}^{n}$, tenemos

$$
\Gamma_{k}(\widetilde{Z})=\left(S^{(0)} \cap S^{(1)} \cap \cdots \cap S^{(k-1)}\right)-S^{(k)}, \quad \forall k \in \mathbb{Z}^{+}
$$

Deseamos observar que el corolario anterior caracteriza los puntos de tangencia de orden $k \geq 2$ en términos de las hiperficies $S^{(0)}, \ldots, S^{(k)}$. Por ejemplo los puntos de tangencia de orden 2 están caracterizados por pertenecer a $T-S^{(1)}$, los puntos de tangencia de orden están caracterizados por pertenecer a $\left(T \cap S^{(1)}\right)-S^{(2)}$, etc.

\section{ESTRUCTURA LOCAL DE LA FOLIACIÓN TRANSFORMADA ESTRICTA ALREDEDOR DE LOS PUNTOS DEL DIVISOR}

De acuerdo a [9], tenemos la siguiente definición.

Definición 5.1 Sea $\beta$ una función satisfaciendo (5). Decimos que $Z \in \mathcal{D}_{\nu}^{n}$ satisface la $\tau$-condición si y sólo si para cada entero $2 \leq s \leq n$, los mapeos

$$
\Phi_{s}=\left(\beta, \widetilde{Z}^{1}(\beta), \ldots, \widetilde{Z}^{s-1}(\beta)\right): \mathbb{C}^{n} \rightarrow \mathbb{C}^{s}
$$

tiene a $0 \in \mathbb{C}^{s}$ como valor regular.

Observación: Si $Z \in \mathcal{D}_{\nu}^{n}$ satisface la $\tau$-condición entonces $\Gamma_{k}(\widetilde{Z})=\emptyset, \forall k \geq n$.

Existe una caracterización de la $\tau$-condición en términos de las hiperficies algebraicas $S^{(j)}$.

Proposición 5.1 Sea $Z \in \mathcal{D}_{\nu}^{n}$, se cumplen las siguienteș afirmaciones:

1. $0 \in \mathbb{C}^{2}$ es un valor regular de $\Phi_{2}=\left(\beta, \widetilde{Z}^{1}(\beta)\right): \mathbb{C}^{n} \rightarrow \mathbb{C}^{2}$ si y sólo si la hiperficie algebraica $S^{(0)}=T$ no tiene puntos singulares.

2. $0 \in \mathbb{C}^{s}(3 \leq s \leq n)$ es un valor regular de $\Phi_{s}=\left(\beta, \widetilde{Z}^{1}(\beta), \ldots, \widetilde{Z}^{s-1}(\beta)\right): \mathbb{C}^{n} \rightarrow \mathbb{C}^{s}$ si y sólo si las hiperficies $S^{(0)}, S^{(1)}, \ldots, S^{(s-2)}$ es transversal.

Prueba. De la definición de las hiperficies algebraicas $S^{(j)}$, se desprende inmediatamente 
que

$$
\Phi_{s}^{-1}(0)=S^{(0)} \cap S^{(1)} \cap \cdots \cap S^{(s-2)}, \quad \forall s \geq 2
$$

Por otro lado, como $\beta(y)=y_{n}$ y $\widetilde{Z}=\sum_{i=1}^{n} \widetilde{Z}_{i} \frac{\partial}{\partial y_{i}}$, entonces

$$
\widetilde{Z}^{1}(\beta)=\sum_{i=1}^{n} \widetilde{Z}_{i} \frac{\partial \beta}{\partial y_{i}}=\widetilde{Z}_{n}
$$

y por tanto la restricción $\left.\widetilde{Z}^{1}(\beta)\right|_{D}=P_{\nu-1}\left(y_{1}, \ldots, y_{n-1}, 1\right)$.

Se sigue que $0 \in \mathbb{C}^{2}$ es un valor regular de $\Phi_{2}=\left(\beta, \widetilde{Z}^{1}(\beta)\right): \mathbb{C}^{n} \rightarrow \mathbb{C}^{2}$ si y sólo si la matriz

$$
\Phi_{2}^{\prime}(p)=\left[\begin{array}{cccc}
0 & \cdots & 0 & 1 \\
\frac{\partial \widetilde{Z}^{1}(\beta)}{\partial y_{1}} & \cdots & \frac{\partial \widetilde{Z}^{1}(\beta)}{\partial y_{n-1}} & \frac{\partial \widetilde{Z}^{1}(\beta)}{\partial y_{n}}
\end{array}\right] .^{(p)}
$$

tiene rango máximo para todo $p \in \Phi_{2}^{-1}(0)=S^{(0)}$ si y sólo si el vector

$$
\left(\frac{\partial \widetilde{Z}^{1}(\beta)}{\partial y_{1}}(p), \ldots, \frac{\partial \widetilde{Z}^{1}(\beta)}{\partial y_{n-1}}(p)\right) \neq(0, \ldots, 0)
$$

para todo $p \in S^{(0)}$ si y sólo si $S^{(0)}$ no tiene puntos singulares. Esto prueba la parte 1.)

Por otro lado, sea $s$ un entero fijo tal que $3 \leq s \leq n$ y sea $1 \leq k \leq s-1$. Tenemos que $0 \in \mathbb{C}^{s}$ es un valor regular de $\Phi_{s}=\left(\beta, \widetilde{Z}^{1}(\beta), \ldots, \widetilde{Z}^{s-1}(\beta)\right): \mathbb{C}^{n} \rightarrow \mathbb{C}^{s}$ si y sólo si la matriz

$$
\Phi_{s}^{\prime}(p)=\left[\begin{array}{cccc}
0 & \cdots & 0 & 1 \\
\frac{\partial \widetilde{Z}^{1}(\beta)}{\partial y_{1}} & \cdots & \frac{\partial \widetilde{Z}^{1}(\beta)}{\partial y_{n-1}} & \frac{\partial \widetilde{Z}^{1}(\beta)}{\partial y_{n}} \\
\vdots & & \vdots & \vdots \\
\frac{\partial \widetilde{Z}^{s-1}(\beta)}{\partial y_{1}} & \cdots & \frac{\partial \widetilde{Z}^{s-1}(\beta)}{\partial y_{n-1}} & \frac{\partial \widetilde{Z}^{s-1}(\beta)}{\partial y_{n}}
\end{array}\right](p)
$$

tiene rango máximo para todo $p \in \Phi_{s}^{-1}(0)$ si y sólo si los vectores

$$
\left\{\left(\frac{\partial \widetilde{Z}^{j}(\beta)}{\partial y_{1}}(p), \ldots, \frac{\partial \widetilde{Z}^{j}(\beta)}{\partial y_{n-1}}(p)\right)\right\}_{1 \leq j \leq n}
$$

son linealmente independientes para todo $p \in \Phi_{s}^{-1}(0)$ si y sólo si $S^{(0)} ; S^{(1)}, \ldots, S^{(s-2)}$ se intersectan transversalmente para todo $p \in \Phi_{s}^{-1}(0)$. 
Proposición $5.2 Z \in \mathcal{D}_{\nu}^{n}$ satisface la $\tau$-condición si y sólo si $S^{(0)}$ no tiene puntos singulares y $S^{(k-2)}$ es transversal a $\left(S^{(0)} \cap S^{(1)} \cap \cdots \cap S^{(k-3)}\right), \forall 3 \leq k \leq n$.

Prueba. Es una consecuencia inmediata de la Proposición (5.1) y la definición de $\tau$ condición.

Proposición 5.3 Genéricamente, las hiperficies $S^{(0)}, S^{(1)}, S^{(j)},(\forall 1 \leq j \leq n-2)$ definidas por el campo vectorial $\widetilde{Z}$, donde $Z \in \mathcal{D}_{\nu}^{n}$, son no singulares.

Prueba. Como sabemos, las hiperficies $S^{(0)}, S^{(1)}, S^{(j)}$ son algebraicas, compactas y son dadas por

$$
S^{(i-1)}=\left\{p=\left[z_{1} ; \ldots ; z_{n}\right] \in D ; \widetilde{Z}^{i}(\beta)(p)=0\right\}
$$

Es suficiente entonces probar la proposición considerando superficies algebraicas definidas por

$$
f\left(z_{1}, \ldots, z_{n}\right)=0
$$

donde $\left(z_{1}, \ldots, z_{n}\right) \in D$.

Observamos que las perturbaciones seran dadas por campos vectoriales en $\mathcal{D}_{\nu}^{n}$, con la finalidad de obtener reultados en $S^{(0)}, S^{(1)}, S^{(j)}$. Sin pérdida de generalidad, podemos suponer que

$$
\bigcap_{i}\left\{\frac{\partial f}{\partial z_{i}}(p)=0\right\}
$$

es un conjunto discreto y compacto. Luego existe $\lambda \in \mathbb{C}, \lambda \neq 0$ con $|\lambda|>0$ suficientemente pequeño, de modo tal que

1. La superficie $f\left(z_{1}, \ldots, z_{n}\right)-\lambda=0$ es no singular.

2. Las superficies $f\left(z_{1}, \ldots, z_{n}\right)=0$ y $f\left(z_{1}, \ldots, z_{n}\right)-\lambda=0$ son cerradas en la topología usual.

Esto finaliza la prueba.

Proposición 5.4 Genéricamente, las hiperficies $S^{(0)}, S^{(1)}, \ldots, S^{(j)},(\forall 1 \leq j \leq n-2)$ se intersectan transversalmente. 
Prueba. Usaremos inducción. Empezamos probando que, genéricamente la intersección entre $S^{(0)}$ y $S^{(1)}$ es transversal.

Por definición, dado $\widetilde{Z}=\sum_{i=1}^{n} \widetilde{Z}_{i} \frac{\partial}{\partial y_{i}}$, tenemos

$$
S^{(0)}=\left\{p \in D ; \widetilde{Z}^{1}(\beta)(p)=0\right\}=\left\{p \in D ; P_{\nu-1}=0\right\}
$$

y

$$
S^{(1)}=\left\{p \in D ; \widetilde{Z}^{2}(\beta)(p)=0\right\}=\left\{p \in D ; \sum_{i=1}^{n} \widetilde{Z}_{i} \frac{\partial P_{\nu-1}}{\partial y_{i}}=0\right\}
$$

$S^{(0)}$ y $S^{(1)}$ son superficies compactas de codimensión uno en $D$. Usando la Proposición (5.3), podemos suponer también que $S^{(0)}$ y $S^{(1)}$ no tienen puntos singulares. Consideremos la intersección $\varphi^{(1)}=S^{(0)} \cap S^{(1)}$ el cual también es compacto en $D$.

Vamos a considerar perturbaciones que preserven $S^{(0)}$. Supongamos, por el absurdo, que $S^{(0)}$ no intersecta transversalmente a $S^{(1)}$. Tomemos $p \in \varphi^{(1)}$, sin pérdida de generalidad podemos suponer que $p=(0, \ldots, 0) \in D,\left(\widetilde{Z}^{1}(\beta)\right)^{\prime}(p)=(1,0, \ldots, 0)$ y $\left(\widetilde{Z}^{2}(\beta)\right)^{\prime}(p)=(\lambda, 0, \ldots, 0)$.

Si denotamos $Z_{\epsilon}=Z+\epsilon z_{2} z_{n}^{\nu} \frac{\partial}{\partial z_{1}}$, entonces un fácil cálculo muestra que $\widetilde{Z}_{\epsilon}=\widetilde{Z}+\epsilon y_{2} \frac{\partial}{\partial y_{1}}$.

Observe que $\widetilde{Z}_{\epsilon}^{1}(\beta)=\widetilde{Z}^{1}(\beta)$ y $\widetilde{Z}_{\epsilon}^{2}(\beta)=\widetilde{Z}^{2}(\beta)+\epsilon y_{2} \frac{\partial \widetilde{Z}^{1}(\beta)}{\partial y_{1}}$. La intersección ahora es $\varphi_{\epsilon}^{(1)}=S^{(0)} \cap S_{\epsilon}^{(1)}$, pero aún $p \in \varphi_{\epsilon}^{(1)},\left(\widetilde{Z}_{\epsilon}^{1}(\beta)\right)^{\prime}(p)=(1,0, \ldots, 0) \mathrm{y}$

$$
\left(\widetilde{Z}_{\epsilon}^{2}(\beta)\right)^{\prime}(p)=\left(\widetilde{Z}^{2}(\beta)\right)^{\prime}(p)+\epsilon \frac{\partial \widetilde{Z}^{1}(\beta)}{\partial y_{1}}(p) \frac{\partial}{\partial y_{2}}=(\lambda, \epsilon, 0, \ldots, 0)
$$

Esto significa que a lo largo de $\left.V_{p}\right|_{\varphi_{\epsilon}^{(1)}}$, vecindad de $p$ restringida a $\varphi_{\epsilon}^{(1)}$, los campos vectoriales $\left(\widetilde{Z}_{\epsilon}^{1}(\beta)\right)^{\prime}$ y $\left(\widetilde{Z}_{\epsilon}^{2}(\beta)\right)^{\prime}$ son linealmente independientes. Como $\varphi_{\epsilon}^{(1)}$ es un conjunto compacto entonces usamos un número finito de tales perturbaciones y obtenemos que $S^{(0)}$ y $S_{\epsilon}^{(1)}$ son transversales.

Supongamos por inducción que la intersección de $S^{(0)}, S^{(1)}, \ldots, S^{(j-1)}(1 \leq j \leq n-2)$ es transversal. Consideremos ahora la superficie no singular $S^{(j)}$, si ella no es transversal a $S^{(0)} \cap \cdots \cap S^{(j-1)}$, denotamos $\varphi^{(j)}=S^{(0)} \cap \cdots \cap S^{(j-1)} \cap S^{(j)}$ el cual es compacto. Si $p \in \varphi^{(j)}$ entonces

$$
\left(\widetilde{Z}^{j+1}(\beta)\right)^{\prime}(p)=\sum_{i=1}^{j} \alpha_{i}\left(\widetilde{Z}^{i}(\beta)\right)^{\prime}(p)
$$


donde $\alpha_{i} \in \mathbb{C}$. Podemos suponer que $p=(0, \ldots, 0) \in D,\left(\widetilde{Z}^{i}(\beta)\right)^{\prime}(p)=\left(a_{1}^{i}, \ldots, a_{j}^{i}, 0, \ldots, 0\right)$, $\widetilde{Z}_{j+1}(p) \neq 0$ y $\frac{\partial \widetilde{Z}_{n}}{\partial y_{i}}(p)=a_{j}^{1} \neq 0,1 \leq i \leq j$.

Denotando $Z_{\epsilon}=Z+\epsilon z_{j+1}^{j} z_{n}^{\nu+1-j} \frac{\partial}{\partial z_{j}}$, entonces $\widetilde{Z}_{\epsilon}=\widetilde{Z}+\epsilon y_{j+1}^{j} \frac{\partial}{\partial y_{j}}$. Observe que también se tiene $\widetilde{Z}_{\epsilon}^{1}(\beta)=\widetilde{Z}^{1}(\beta)$ y $\widetilde{Z}_{\epsilon}^{2}(\beta)=\widetilde{Z}^{2}(\beta)+\epsilon y_{j+1}^{j} \frac{\partial \widetilde{Z}_{n}}{\partial y_{1}}$ y

$$
\widetilde{Z}_{\epsilon}^{k}(\beta)=\widetilde{Z}^{k}(\beta)+\epsilon y_{j+1}^{(j+1-(k-2)} C_{k}^{j+1}+\frac{j !}{(k-2) !} \epsilon \widetilde{Z}_{j+1}^{j-(k-2)} y_{j+1}^{j-(k-2)} \frac{\partial \widetilde{Z}_{n}}{\partial y_{j}}, \quad 1 \leq k \leq j+1
$$

donde $C_{k}^{j+1}$ son funciones analíticas. La intersección ahora es

$$
\varphi_{\epsilon}^{(j)}=S^{(0)} \cap S_{\epsilon}^{(1)} \cap \cdots \cap S_{\epsilon}^{(j)}
$$

la cual aún continua pasando por $p$. Note que se tiene

$$
\left(\widetilde{Z}_{\epsilon}^{k}(\beta)\right)^{\prime}(p)=\left(\widetilde{Z}^{k}(\beta)\right)^{\prime}(p), \quad 1 \leq k \leq j
$$

$\mathrm{y}$

$$
\left(\widetilde{Z}_{\epsilon}^{j+1}(\beta)\right)^{\prime}(p)=\left(\widetilde{Z}^{j+1}(\beta)\right)^{\prime}(p)+j \epsilon \widetilde{Z}_{j+1}(p) \frac{\partial \widetilde{Z}_{n}}{\partial y_{j}}(p) \frac{\partial}{\partial y_{j+1}}
$$

Esto significa que a lo largo de $\left.V_{p}\right|_{\varphi_{\epsilon}^{(j)}}$, vecindad de $p$ restringida a $\varphi_{\epsilon}^{(j)}$, los campos vectoriales $\left(\widetilde{Z}_{\epsilon}^{1}(\beta)\right)^{\prime}, \ldots,\left(\widetilde{Z}_{\epsilon}^{j+1}(\beta)\right)^{\prime}$ son linealmente independientes. Como $\varphi_{\epsilon}^{(1)}$ es un conjunto compacto entonces usamos un número finito de tales perturbaciones y obtenemos que la intersección $S^{(0)} \cap \cdots \cap S_{\epsilon}^{(j)}$ es transversal. Esto finaliza la demostración.

Denotaremos por $\mathcal{D}_{\nu}^{n}(\tau)$ al conjunto de todos los campos vectoriales holomorfos $Z \in \mathcal{D}_{\nu}^{n}$ que satisfacen la $\tau$-condición.

Teorema $5.5 \mathcal{D}_{\nu}^{n}(\tau)$ es abierto y denso en $\mathcal{D}_{\nu}^{n}$.

Prueba. Es claro que $\mathcal{D}_{\nu}^{n}(\tau)$ es un subconjunto abierto de $\mathcal{D}_{\nu}^{n}$. La densidad es consecuencia de las Proposiciones (5.3) y (5.4).

Observación: El Teorema (5.5) nos dice que todo campo vectorial $Z \in \mathcal{D}_{\nu}^{n}$ puede ser 
perturbado de tal manera que su hiperficie de tangencia sea no singular (en particular irreducible) y tal que sólo existan puntos con orden de tangencia $1,2, \ldots, n$. Más aún, todos los puntos de orden de tangencia $j(2 \leq j \leq n)$ están caracterizados por pertenecer a intersecciones transversale de hiperficies algebraicas.

Finalmente, exhibir formas normales locales de todos los puntos $p \in D$ de un campo vectorial holomorfo que satisface la $\tau$-condición. Como es de imaginarse, esta forma local depende del orden de tangencia.

Teorema 5.6 Sea $Z \in \mathcal{D}_{\nu}^{n}(\tau)$. Si $p_{0} \in \Gamma_{k}(\widetilde{Z})$, entonces podemos encotrar coordenadas locales $t, x_{1}, \ldots, x_{n-1}$ en una vecindad $\tilde{U}$ de $p_{0}$, que se anula en $p_{0}$ tal que

1. $\left.\widetilde{Z}\right|_{\tilde{U}}=\frac{\partial}{\partial t}$

2. $D \cap \tilde{U}$ es dado por $\widetilde{\gamma}^{-1}(0)$ donde

$$
\widetilde{\gamma}=(t(p))^{k}+\sum_{j=1}^{k-1} x_{j}(p)(t(p))^{j-1}
$$

En el caso real, el Teorema (5.6) fue probado independientemente por Percell (ver [9]) y Sotomayor (ver [11]) usando el Teorema de Preparación de Malgrange-Mather. En nuestro caso, la prueba del teorema se sigue del Teorema de Preparación de Weierstrass.

Observación: Cuando $Z \in \mathcal{D}_{\nu}^{3}(\tau)$, del teorema anterior se tienen las siguientes posibilidades:

1. Si $p_{0} \in \Gamma_{0}(\widetilde{Z})$ entonces el divisor $D$ en la vecindad $D \cap \widetilde{U}$ de $p_{0}$ es dado por

$$
D \cap \widetilde{U}=\left\{\left(t, x_{1}, x_{2}\right) ; t=0\right\}
$$

2. Si $p_{0} \in \Gamma_{1}(\widetilde{Z})$ entonces

$$
D \cap \widetilde{U}=\left\{\left(t, x_{1}, x_{2}\right) ; t^{2}+x_{1}=0\right\}
$$

3. Si $p_{0} \in \Gamma_{2}(\widetilde{Z})$ entonces

$$
D \cap \widetilde{U}=\left\{\left(t, x_{1}, x_{2}\right) ; t^{3}+x_{1}+x_{2} t=0\right\}
$$


En todos los casos, en la vecindad $\widetilde{U}$ de $p_{0}$ la foliación $\widetilde{\mathcal{F}}_{Z}$ viene es la inducida por el campo constante $(1,0,0)$. Esto nos da un comportamiento geométrico local satisfactorio en la vecindad de cada punto del divisor.

\section{REFERENCIAS}

[1] R. Benazic - A Resolution Theorem for Absolutely Isolated Singularities of Holomorphic Vector Fields, Bol. Soc. Bras. Mat., Vol. 28, N 1, pp. 211-231.

[2] R. Benazic - Singularidades dicríticas de Campos Vectoriales Complejos, Fondo Editorial UNMSM, Lima (2002).

[3] R. Benazic - Tópicos de Ecuaciones Diferenciales Ordinarias, Universidad Nacional de Ingeniería, Lima (2007).

[4] M. I. Camacho - On the Local Structure of Real Vector Fields at a Dicritical Singularity, Preprint IMPA (1997).

[5] F. Cano - Final forms for a 3-dimensional vector field under blowing-up, Ann. Inst. Fourier 3, 2 (1987), pp. 151-193.

[6] C. Camacho, F. Cano, P. Sad - Absolutely isolated Singularities of Holomorphic Vector Fields, Invent. Math. 98, (1989), pp. 351-369.

[7] E. Chirka - Complex Analytic Sets, MIA, Kluwer Academic Publishers. Dordrecht, Boston, London (1989).

[8] M. Klughertz - Feuilletages holomorphes a singularité isolle ayant une infinite de courbes intégrales, These, Université Paul Sabatier - Toulouse, (1988).

[9] P. Percell - Structural Stability on Manifolds with Boundary, Topology, vol. 12 (1973).

[10] A. Seidenberg - Reduction of singularities of the differentiable equation $A d y=B d x$, Amer. J. Math. 90 (1968), pp. 248-269.

[11] J. Sotomayor - Structural Stability in Manifolds with Boundary, Global Analysis and its applications, vol. III, International Atomic Energy Agency, Vienna (1974).

[12] J. Sotomayor - Lições de Equações Diferenciais Ordinarias, Projeto Euclides, IMPA (1979).

[13] A. Ven Den Essen - Reduction of singularities of the differentiable equation $A d y=B d x$, Lectures Notes in Mathematics, Vol. 712, pp. 44-59. 